\title{
Obstetric and neonatal outcomes in women with epilepsy in Poland - a two-centre study
}

\author{
Magdalena Bosak ${ }^{1}$, Bryan H. Song ${ }^{2}$, Milena Dewerenda-Sikora ${ }^{3}$, Agnieszka Słowik ${ }^{1}$, Anetta Lasek-Bal ${ }^{3}$ \\ ${ }^{1}$ Department of Neurology, Jagiellonian University Medical College, Krakow, Poland \\ ${ }^{2}$ School of Medicine in English, Jagiellonian University Medical College, Krakow, Poland \\ ${ }^{3}$ Department of Neurology, School of Health Sciences, Medical University of Silesia in Katowice, Poland
}

\begin{abstract}
Aim of the study. To report pregnancy, delivery and perinatal outcomes in women with epilepsy seen in university clinics in Poland. Clinical rationale for the study. Women with epilepsy are reported to be at increased risk of adverse pregnancy and foetal outcomes.

Materials and methods. The following data was collected in 171 pregnant women seen in two university epilepsy clinics: epilepsy characteristics and treatment, seizure frequency, pregnancy outcomes, perinatal outcomes, and feeding method.

Results. The mean age of patients at the time of delivery was 28.8 years, and most women were nulliparous. Almost $80 \%$ of patients were on monotherapy and the majority were prescribed antiepileptic drugs with low teratogenic potential. $53.8 \%$ of patients were seizure-free during pregnancy. Half of the cohort delivered by caesarean section and the majority delivered in term. Nearly all newborns scored $>7$ Apgar points. Major congenital malformation was diagnosed in only one foetus. Any adverse pregnancy/neonatal outcome was found in $13 \%$ of patients. Two thirds of women reported breastfeeding at any time.

Conclusions and clinical implications. Almost $90 \%$ of women continued antiepileptic therapy during pregnancy. $46 \%$ of patients had epileptic seizures during pregnancy. Any adverse pregnancy/neonatal outcome was found in $13 \%$ of women with epilepsy. The majority of patients delivered healthy babies. Further studies are needed to find risk factors for adverse pregnancy/ neonatal outcomes in women with epilepsy in Poland.
\end{abstract}

Key words: epilepsy, women, pregnancy, delivery, perinatal outcomes

(Neurol Neurochir Pol 2020; 54 (1): 62-65)

\section{Introduction}

Epilepsy affects about $0.5 \%$ of women of reproductive age $[1,2]$. To avoid the maternal and foetal risks associated with seizures, most women with epilepsy (WWE) require ongoing medical therapy with antiepileptic drugs (AEDs) during pregnancy. WWE, especially those treated with AEDs, are reported to be at increased risk of adverse pregnancy and foetal outcomes [3, 4]. Various obstetric complications such as caesarean section, miscarriage, preterm birth, antepartum and postpartum haemorrhage, and maternal death have been reported more frequently in WWE than in the general population [5]. Exposure to AEDs and seizures in utero may increase the rates of congenital malformations, neurodevelopmental problems, growth restriction and perinatal death in children of WWE $[4,6]$.

The results of many prospective and retrospective registers have been published, improving our understanding of risks in pregnancies and babies of WWE. However, there is a significant variation in reported data across countries, ethnicities, geographical locations and socioeconomic status. Thus, each population group should have its own data on pregnancy and neonatal outcomes among WWE. 


\section{Clinical rationale for the study}

The aim of this study was to report pregnancy, delivery and perinatal outcomes in women with epilepsy seen in university clinics in Poland.

\section{Material and methods}

This study had a cross-sectional design and was carried out in two university hospitals in Poland. Data from pregnant WWE was collected prospectively from 1 January 2012 and 30 June 2018 in one university hospital. Data from the same time period was retrieved retrospectively from medical records of WWE treated in a second university hospital.

The following data was collected: age at delivery, age at onset of epilepsy, epilepsy and seizure type, seizure frequency before (12 months) and during pregnancy, epilepsy treatment during pregnancy, AEDs dosage changes in pregnancy, and maternal preexisting chronic conditions. Pregnancy outcomes examined in this study were as follows: gestational diabetes, gestational hypertension, preeclampsia, ante- and postpartum haemorrhage, and mode of delivery. Perinatal outcomes included stillbirth, preterm birth, spontaneous or medically induced abortion, small-for-gestational-age (SGA) live births, 5-minute Apgar score, and the presence of major congenital malformation (MCM) at birth. Feeding method (breast- or bottle-feeding) was also noted, with information obtained 3-6 months after delivery.

We defined an adverse pregnancy and/or perinatal outcome as the occurrence of any of the following: gestational diabetes, gestational hypertension, preeclampsia, ante- and postpartum haemorrhage, status epilepticus in pregnancy, preterm birth, spontaneous or medically induced abortion, low birth weight $(<2,500 \mathrm{~g}), 5$-minute Apgar score $<7$, and the presence of congenital malformation at birth.

This study was approved by local ethics committees and complied with the principles of the Declaration of Helsinki.

\section{Statistical analysis}

Frequencies were reported as numbers and percentages, continuous data as means with standard deviations and minimum/maximum as appropriate. The significance of the differences between groups was tested with $\chi 2$ for categorical variables or with Mann-Whitney U-test for continuous data. Predictors of the occurrence of adverse pregnancy/neonatal outcomes were assessed with univariate analysis. Calculations were performed using a Statistica 13.1 package.

\section{Results}

171 WWE, who were pregnant between 1 January 2012 and 30 June 2018, were enrolled in the study. The mean age of the patients at delivery was 28.8 years (range 19-41). Focal epilepsy was diagnosed in 108 patients (63.2\%). Epilepsy was diagnosed in two patients during pregnancy (8th and 9th month of pregnancy). Most women were nulliparous (116, 67.8\%). One patient suffered from hereditary peripheral neuropathy, one from a dysembryoplastic neuroepithelial tumour (surgery five years before pregnancy), and one underwent a subarachnoid haemorrhage with the clipping of multiple aneurysms.

AEDs were prescribed in the majority of pregnancies $(158,92.4 \%)$, mainly in monotherapy. The most frequently used AEDs were levetiracetam (LEV) and lamotrigine (LTG). In 52 women (32.7\% of those receiving treatment) doses of AEDs were increased in pregnancy (19 LTG, $18 \mathrm{LEV}$, seven carbamazepine (CBZ), two valproate (VPA), and in six a new AED was added to the therapy regimen. One patient with epilepsy onset in the first trimester developed a rash related to $\mathrm{CBZ}$ and was switched from CBZ to LEV.

More than half of WWE $(99,57.9 \%)$ were seizure-free during the 12 months prior to conception; 92 (53.8\%) remained so throughout pregnancy. One case of nonconvulsive status epilepticus occurred in the second trimester, and this was possibly related to the tapering of AEDs before conception.

Half of the cohort $(83,49.7 \%)$ delivered by caesarean section, and the majority delivered in term $(156,91.3 \%)$. There were four miscarriages, one induced abortion due to congenital malformations, and one stillbirth among the studied patients. One patient had gestational hypertension, and two had antepartum haemorrhages.

According to the Apgar score, the majority of newborns were $>7$ points. Two offspring $(0.8 \%)$ scored $<7$ points, and they were exposed in utero to VPA or CBZ. One foetus was diagnosed with multiple MCMs and a medically induced abortion was performed.

The detailed characteristics of patients in terms of epilepsy, its treatment, pregnancy and neonatal outcomes are set out in Table 1.

$111(68.1 \%)$ of the WWE reported breastfeeding at any time. Any adverse pregnancy/neonatal outcome was present in $22(12.9 \%)$ of the WWE. Neonatal adverse outcomes included four miscarriages, one MCM, one stillbirth, two Apgar score $<7$ points, and one Down's syndrome. Pregnancy adverse outcomes were as follow: gestational hypertension (1), haemorrhage (2), live preterm birth (9), and status epilepticus in pregnancy (1).

We compared patients with an adverse pregnancy and/or perinatal outcome in terms of their age, age at onset of epilepsy, epilepsy type, seizure occurrence during pregnancy and the pre-pregnancy year, epilepsy therapy in pregnancy (number and types of AEDs), AEDs dose increase in pregnancy, gravidity, and parity. No variables were statistically significant in univariate analysis.

\section{Discussion}

To the best of our knowledge, this is the first study reporting pregnancy and perinatal outcomes in Polish WWE. Any 
Table 1. Demographic and clinical characteristics of subjects

\begin{tabular}{|c|c|}
\hline \multicolumn{2}{|l|}{ Variable } \\
\hline Age at delivery (years); \pm SD [range] & $28.8 \pm 4.6[19-41]$ \\
\hline Age at onset of epilepsy (years); \pm SD [range] & $15.1 \pm 7.2[2-38]$ \\
\hline \multicolumn{2}{|l|}{ Epilepsy type; n (\%) } \\
\hline Generalised & $63(36.8)$ \\
\hline Focal & $108(63.2)$ \\
\hline \multicolumn{2}{|l|}{ Seizures in pregnancy; $\mathbf{n}(\%)$} \\
\hline Yes & $79(46.2)$ \\
\hline No & $92(53.8)$ \\
\hline \multicolumn{2}{|l|}{ Seizures during pre-pregnancy year; $\mathbf{n}(\%)$} \\
\hline Yes & $72(42.1)$ \\
\hline No & 99 (57.9) \\
\hline \multicolumn{2}{|l|}{ Epilepsy therapy in pregnancy; $\mathbf{n}(\%)$} \\
\hline No AEDs & $13(7.6)$ \\
\hline Monotherapy & $133(77.8)$ \\
\hline Polytherapy & $25(14.6)$ \\
\hline \multicolumn{2}{|l|}{$\begin{array}{l}\text { Most commonly used AEDs (in mono- or } \\
\text { polytherapy); } \mathbf{n}(\%)\end{array}$} \\
\hline Lamotrigine & $46(26.9)$ \\
\hline Levetiracetam & $42(24.6)$ \\
\hline Valproate & $28(16.4)$ \\
\hline Carbamazepine & $19(11.1)$ \\
\hline \multicolumn{2}{|l|}{ Gravidity; n (\%) } \\
\hline 1 & $105(61.4)$ \\
\hline 2 & $51(29.8)$ \\
\hline 3 & $14(8.2)$ \\
\hline 5 & $1(0.6)$ \\
\hline \multicolumn{2}{|l|}{ Parity, n (\%) } \\
\hline 1 & $116(67.8)$ \\
\hline 2 & $46(26.9)$ \\
\hline 3 & $8(4.7)$ \\
\hline 4 & $1(0.6)$ \\
\hline \multicolumn{2}{|l|}{ Live birth; $\mathbf{n}(\%)$} \\
\hline Yes & $165(96.5)$ \\
\hline No & $6(3.5)$ \\
\hline \multicolumn{2}{|l|}{ Gestational age; $\mathbf{n}(\%)$} \\
\hline Miscarriage or abortion ( $\leq 20$ weeks) & $5(2.9)$ \\
\hline Preterm birth (21-36) & $10(5.8)$ \\
\hline Term birth (37-42) & $156(91.3)$ \\
\hline \multicolumn{2}{|l|}{ Delivery method; n (\%) } \\
\hline Vaginal delivery & $82(50.3)$ \\
\hline Caesarean section & $83(49.7)$ \\
\hline \multicolumn{2}{|l|}{ Apgar score; n (\%) } \\
\hline$<7$ & $2(0.8)$ \\
\hline$\geq 7$ & $163(99.2)$ \\
\hline \multicolumn{2}{|l|}{ Feeding method; n (\%) } \\
\hline Breast feeding & $111(68.1)$ \\
\hline Bottle feeding & $52(31.9)$ \\
\hline
\end{tabular}

SD — standard deviation; AED — antiepileptic drug adverse pregnancy/neonatal outcome was found in $22(12.9 \%)$ of the WWE.

Most patients (87\%) were on monotherapy and the majority were prescribed AEDs with low teratogenic potential (LEV, LTG, OXC). This is in line with the recommendations for pregnant WWE $[7,8] .53 .8 \%$ of the WWE were seizure-free during pregnancy, and this percentage is only slightly lower than in the recent study from EURAP [9]. In 52 women, doses of AEDs were increased in pregnancy, with most of them treated with LEV or LTG. This finding is concordant with other studies and results from pharmacokinetic changes of both AEDs in pregnancy $[10,11]$.

Malformation rate in this cohort was very low $(0.6 \%)$, MCM was detected only in one foetus, and resulted in induced abortion. This finding may be related to the low rate of exposure to highly teratogenic AEDs. However, newborns were not followed later, and data on malformation detected at the age of 12 months was not available.

Half of our cohort delivered through caesarean section. This rate is much higher than in other studies [5]. This finding may be related to the similarly high percentage of caesarean sections in the general Polish population [12]. The high rate of caesarean section in the WWE is discordant with the recent guidelines of the Polish Society of Epileptology and the Polish Gynaecological Society [13]. Only one patient (treated with VPA) had gestational hypertension. However, the WWE, particularly those receiving VPA, were found to be at increased risk of hypertensive pregnancy complications [14].

Due to the numerous health benefits for infants and mothers, breastfeeding should be recommended for all women, including patients with epilepsy [13]. Recent studies $[15,16]$. Have found higher IQ, cognitive abilities and no adverse effects in breastfed children of WWE treated with AEDs. Two thirds of WWE reported breastfeeding at any time, and this rate is similar to other studies [17].

We were not able to find any risk factors for adverse pregnancy/neonatal outcomes in WWE, which may be related to the small number of patients included and the partly retrospective design of the study.

We acknowledge several limitations related to our study. Firstly, data was collected prospectively only in $62.5 \%$ of pregnancies. The partly retrospective design of the study could result in some missing significant information. Secondly, the cohort of studied patients involved women seen in a tertiary epilepsy clinic, and may not be representative of the general population of female patients with epilepsy in terms of seizure frequency and epilepsy treatment. Thirdly, our study did not contain a control group.

\section{Conclusions}

Almost 90\% of women continued antiepileptic therapy during pregnancy. $46 \%$ of patients had epileptic seizures during pregnancy. Any adverse pregnancy/neonatal outcome 
was found in $13 \%$ of women with epilepsy. The majority of patients delivered healthy babies. Further studies are needed to find risk factors for adverse pregnancy/neonatal outcomes in Polish women with epilepsy.

\section{Acknowledgments:}

The authors received no funding for this work.

\section{Declaration of interest:}

MB received honoraria for publications/participation in advisory meetings from Sanofi; honoraria for lectures and conference fees from Sanofi, Adamed, Teva, Neuraxpharm.

$\mathrm{BS}$ and MDS have nothing to declare.

AS received honoraria for lectures from Bayer, Boehringer, Novartis, Polpharma, Bristol-Myers, Biogen, Medtronic; for participation in advisory meetings from Bayer, Boehringer, Novartis.

ALB received honoraria for lectures/publications from Bayer, Boehringer, Novartis, Balton, Biofarm, Biogen, for participation in advisory meetings from Bayer, Merck.

\section{References:}

1. Jette N, Fiest KM, Sauro KM, et al. Prevalence and incidence of epilepsy: A systematic review and meta-analysis of international studies. Neurology. 2017; 88(3): 296-303, doi: 10.1212/ WNL.0000000000003509, indexed in Pubmed: 27986877.

2. Wallace $H$, Shorvon $S$, Tallis R. Age-specific incidence and prevalence rates of treated epilepsy in an unselected population of 2,052,922 and age-specific fertility rates of women with epilepsy. Lancet. 1998; 352(9145): 1970-1973, doi: 10.1016/S0140-6736(98)04512-7, indexed in Pubmed: 9872246.

3. Razaz N, Tomson T, Wikström AK, et al. Association Between Pregnancy and Perinatal Outcomes Among Women With Epilepsy. JAMA Neurol. 2017; 74(8): 983-991, doi: 10.1001/jamaneurol.2017.1310, indexed in Pubmed: 28672292.

4. Tomson T, Battino D, Bonizzoni E, et al. EURAP Study Group. Comparative risk of major congenital malformations with eight different antiepileptic drugs: a prospective cohort study of the EURAP registry. Lancet Neurol. 2018; 17(6): 530-538, doi: 10.1016/S1474-4422(18)301078, indexed in Pubmed: 29680205.

5. Viale L, Allotey J, Cheong-See F, et al. EBM CONNECT Collaboration. Epilepsy in pregnancy and reproductive outcomes: a systematic review and meta-analysis. Lancet. 2015; 386(10006): 1845-1852, doi: 10.1016/S0140-6736(15)00045-8, indexed in Pubmed: 26318519.

6. Meador KJ, Baker GA, Browning N, et al. NEAD Study Group. Fetal antiepileptic drug exposure and cognitive outcomes at age 6 years
(NEAD study): a prospective observational study. Lancet Neurol. 2013; 12(3): 244-252, doi: 10.1016/S1474-4422(12)70323-X, indexed in Pubmed: 23352199.

7. Harden CL, Meador KJ, Pennell PB, et al. American Academy of Neurology, American Epilepsy Society. Management issues for women with epilepsy-Focus on pregnancy (an evidence-based review): II. Teratogenesis and perinatal outcomes: Report of the Quality Standards Subcommittee and Therapeutics and Technology Subcommittee of the American Academy of Neurology and the American Epilepsy Society. Epilepsia. 2009; 50(5): 1237-1246, doi: 10.1111/j.1528-1167.2009.02129.x, indexed in Pubmed: 19507301.

8. Patel SI, Pennell PB. Management of epilepsy during pregnancy: an update. Ther Adv Neurol Disord. 2016; 9(2): 118-129, doi: 10.1177/1756285615623934, indexed in Pubmed: 27006699.

9. Battino D, Tomson T, Bonizzoni E, et al. EURAP Study Group. Seizure control and treatment changes in pregnancy: observations from the EURAP epilepsy pregnancy registry. Epilepsia. 2013; 54(9): 16211627, doi: 10.1111/epi.12302, indexed in Pubmed: 23848605.

10. Pennell PB, Peng L, Newport DJ, et al. Lamotrigine in pregnancy: clearance, therapeutic drug monitoring, and seizure frequency. Neurology. 2008; 70(22 Pt 2): 2130-2136, doi: 10.1212/01. wnl.0000289511.20864.2a, indexed in Pubmed: 18046009.

11. Vajda FJE, O'Brien T, Lander C, et al. The efficacy of the newer antiepileptic drugs in controlling seizures in pregnancy. Epilepsia. 2014; 55(8): 1229-1234, doi: 10.1111/epi.12711, indexed in Pubmed: 24995555.

12. http://www.imid.med.pl/files/imid/Aktualnosci/Aktualnosci/ Dane\%20statystyczne\%20EURO-PERISTAT\%20+\%20IMiD\%20+\%20 GUS.pdf (01 March 2019).

13. Jędrzejczak J, Bomba-Opoń D, Jakiel G, et al. Managing epilepsy in women of childbearing age - Polish Society of Epileptology and Polish Gynecological Society Guidelines. Ginekol Pol. 2017; 88(5): 278-284, doi: 10.5603/GP.a2017.0053, indexed in Pubmed: 28580576.

14. Danielsson KC, Borthen I, Morken NH, et al. Hypertensive pregnancy complications in women with epilepsy and antiepileptic drugs: a population-based cohort study of first pregnancies in Norway. BMJ Open. 2018; 8(4): e020998, doi: 10.1136/bmjopen-2017-020998, indexed in Pubmed: 29691249.

15. Meador KJ, Baker GA, Browning N, et al. Neurodevelopmental Effects of Antiepileptic Drugs (NEAD) Study Group, NEAD Study Group. Effects of breastfeeding in children of women taking antiepileptic drugs. Neurology. 2010; 75(22): 1954-1960, doi: 10.1212/ WNL.0b013e3181ffe4a9, indexed in Pubmed: 21106960.

16. Meador KJ, Baker GA, Browning N, et al. Neurodevelopmental Effects of Antiepileptic Drugs (NEAD) Study Group. Breastfeeding in children of women taking antiepileptic drugs: cognitive outcomes at age 6 years. JAMA Pediatr. 2014; 168(8): 729-736, doi: 10.1001/jamapediatrics.2014.118, indexed in Pubmed: 24934501.

17. Johnson EL, Burke AE, Wang A, et al. Unintended pregnancy, prenatal care, newborn outcomes, and breastfeeding in women with epilepsy. Neurology. 2018; 91(11): e1031-e1039, doi: 10.1212/ WNL.0000000000006173, indexed in Pubmed: 30097479. 